NASA

\title{
THE FOKKER-PLANCK COEFFICIENT FOR PITCH-ANGLE SCATTERING OF COSMIC RAYS -
}

\author{
L. A. FISK \\ M. L. GOLDSTEIN \\ A. J. KLIMAS \\ G. SANDRI
}

究

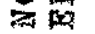

叫 Q 1 ind

10 3

国 00 on 2 跫是 e U

E

$\rightarrow$ en in 0 P i

a en $\therefore \geq$ 1 is

政然

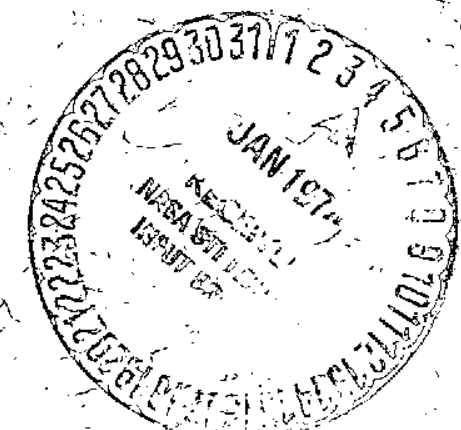


The Fokker-Planck Coefficient for Pitch-Angle Scattering of Cosmic Rays

$$
\text { by }
$$

\author{
I. A. Fisk \\ Laboratory for High Energy Astrophysics \\ NASA, Goddard Space Flight Center \\ Greenbe1t, Maryland 20771
}

M. L. Goldstein* and A. J. K1imas**

Laboratory for Extraterrestrial Physics

NASA, Goddard Space Flight Center

Greenbe1t, Maryland 20771

\title{
G. Sandri
}

Aeronautical Research Associates of Princeton, Inc. Princeton, New Jersey 08540

* NAS-NRC Postdoctoral Resident Research Associate

** NAS-NRC Senior Postdoctoral Resident Research Associate 
For the case of homogeneous, isotropic magnetic field fluctuations, it is shown that most theories which are based on the quasi-1inear and adiabatic approximations yield the same integral for the Fokker-Planck coefficient for the pitch angle scattering of cosmic rayso For example, despite apparent differences, the theories due to Jokipii and to Klimas and Sandri yield the same integral. It is also shown, however, that this integral in most cases has been evaluated incorrectly in the past. For small pitch angles, the errors in previous evaluations are fortuitously of minor importance. For large pitch angles, however, these errors become more significant, and for pitch angles of $90^{\circ}$, the actual Fokker-Planck coefficient contains a delta-function which has been overlooked in the past. The implications for these corrections on our ability to relate cosmic-ray diffusion coefficients to observed properties of the interplanetary magnetic field are discussed.

$$
i j
$$




\section{Introduction}

In recent years a number of seemingly different theories have been developed for relating the cosmic-ray diffusion tensor to observed properties of the interplanetary magnetic field (e.g. Jokipii, 1966, 1967, 1968, 1971, 1972; Hal1 and Sturrock, 1967; Hasselmann and Wibberenz, 1968; Klimas and Sandri, 1971, 1973a,b). These theories are derived by using versions of both the quasi-linear and the adiabatic approximations. In the quasi-linear approximation the inter action between the particles and the random magnetic field is assumed to be sufficiently weak so that to lowest order of interest a particle executes an unperturbed helix about the mean field while interacting with the random field. In the adiabatic approximation the cosmic-ray distribution function is assumed to vary on a time scale which is longer than and distinct from the time scale over which a particle experiences a significant interaction with the random magnetic field (see Appendix A to this paper for details concerning how these approximations are used, and for conditions under which they are valid).

In this paper we show that despite apparent differences there are substantial areas of agreement between the various theories. For example, we show that in the case of magnetic field fluctuations that are homogeneous and isotropic the theories due to Jokipii (1966) and to Klimas and Sandri (1971, 1973a,b) yie1d the same basic integral for calculating the Fokker-P1anck coefficient for pitch angle scattering $\left(<\left(\Delta_{\mu}\right)^{2}\right\rangle / \Delta t$, where $\mu$ is the cosine of a particle's pitch angle). We also point out, however, that this integral for $\left\langle(\Delta \mu)^{2}\right\rangle / \Delta t$ in 
most cases has been evaluated incorrectly in the past. For example, there is no formal justification for the approximations due to Jokipii (1966, 1971), which permit this integral to be expressed simply in terms of a one-dimensional power spectrum of magnetic field fluctuations, evaluated at a resonant wavenumber. By using both analytic and numerical techniques, we correctly evaluate $\left\langle\left(\Delta_{\mu}\right)^{2}\right\rangle / \Delta t$ for a general form of the power spectrum, which should provide a good fit to observed power spectra. In the limit of low rigidity, where the ratio of the correlation length for field fluctuations to the particle's gyro-radius is large, the correct result for $\left\langle(\Delta \mu)^{2}\right\rangle / \Delta t$ differs from that due to Jokipii (1966) by a factor of $|\mu|($ for $\mu \neq 0)$. At a11 but very small values of $\mu$, this correction will introduce only a small numerical change in predicted values of $\left\langle(\Delta \mu)^{2}\right\rangle / \Delta t$. However, in certain schemes for averaging $<\left(\Delta_{\mu}\right)^{2}>/ \Delta$ t over $\mu$, and for forming the diffusion coefficient for propagation parallel to the mean fleld (Jokipii, 1966, 1971; Ear1, 1973), this extra factor of $|\mu|$ introduces strong divergences as $\mu \rightarrow 0$. These divergences no doubt result from the inadequacies near $\mu=0$ of the quasi-linear and adiabatic approximations. We also show that quasi-linear/adiabatic theory does not in general predict that $\left.<\left(\Delta_{\mu}\right)^{2}\right\rangle / \Delta t=0$ at $\mu=0$ (e.g. Jokipii, 1966, 1971) or that $\left\langle(\Delta \mu)^{2}\right\rangle / \Delta t$ diverges continuously to infinity as $\mu \rightarrow 0$ (Hasselmann and Wibberenz, 1968). Rather, this theory predicts for physically realistic power spectra that $\left\langle(\Delta \mu)^{2}\right\rangle / \Delta t$ contains a delta-function, $\delta(\mu)$. Finally, we discuss how the above corrections affect our ability to determine an accurate diffusion coefficient for propagation parallel to the mean 
field, and how they affect our ability to eliminate the inadequacies in current quasi-1inear/adiabatic theory.

Agreement Among the Theories

The formula for $\left\langle(\Delta \mu)^{2}\right\rangle / \Delta t$ due to Jokipii (1966) contains a minor algebraic mistake. In Appendix A to this paper we rederive the correct expression for $\left\langle(\Delta \mu)^{3}\right\rangle / \Delta$ t that results from applying the quasilinear and adiabatic approximations. We follow here procedures very similar to those used by Jokipii (1966). The resulting $\left\langle(\Delta \mu)^{2}\right\rangle / \Delta t$ for homogeneous, isotropic field fluctuations, which is the case considered commonly by Jokipii (1966) and K1imas and Sandri (1971, 1973a,b), is given by

$\frac{<(\Delta \mu)^{2} \geq}{\Delta t}=\frac{\left(1-\mu^{2}\right) \eta^{2} \varepsilon^{2} v}{\lambda_{c}} \int_{0}^{\infty} \mathrm{d} \lambda\left[\cos (\varepsilon \lambda)\left[2 b\left(r / \lambda_{c}\right)+\rho^{2} a\left(r / \lambda_{c}\right)\right]-\rho^{2} a\left(r / \lambda_{c}\right)\right]$

Here $\epsilon=\lambda_{c} / r_{g}$, the ratio of the correlation length of the field fluctuations to the particle's gyro-radius; $v$ is particle speed; and $\eta^{2}=\mathrm{B}_{\mathrm{rms}}^{2} / \mathrm{B}_{\mathrm{O}}^{2}$ is the ratio of the mean square field strength to the mean field strength squared. The functions $a$ and $b$ of the scaler quantity $r / \lambda_{c}$ are the arbitrary functions that appear in the two-point correlation tensor for homogeneous, isotropic fluctuations (Batchelor, 1956):

$$
\begin{aligned}
C_{i j}(\underline{r}) & \equiv\left\langle B_{i}(\underline{R}) B_{j}(\underline{R}+\underline{r})>/ \eta^{2} B_{o}^{2}\right. \\
& =a\left(r / \lambda_{c}\right) r_{i} r_{j}+b\left(r / \lambda_{c}\right) \delta_{i j}
\end{aligned}
$$

The functions $a$ and $b$ in (1) are to be evaluated at 
$r / \lambda_{c}=\left[\mu^{2} \lambda^{2}+\frac{2\left(1-\mu^{2}\right)}{\epsilon^{2}}(1-\cos (\epsilon \lambda))\right]^{\frac{1}{2}} \equiv\left[\mu^{2} \lambda^{2}+\rho^{2}\right]^{\frac{1}{2}}$

which is the linear distance from a point in a particle's helical orbit along the mean field (chosen to be the origin) to the particle's position at a dimensionless time $\lambda=v t / \lambda_{c}$ later.

In the expression for $\left\langle\left(\Delta_{\mu}\right)^{2}\right\rangle / \Delta$ t due to Jokipii (cf. equation (18) in Jokipii (1966) or equation (32) in Jokipii (1971)) the sign of the second term containing $a\left(r / \lambda_{c}\right)$ in ( 1 ) is incorrectly positive. Jokipii $(1966,1971)$ subsequent $1 y$ neglects these terms containing $a\left(r / \lambda_{c}\right)$ so that the formulas for $\left\langle\left(\Delta_{H}\right)^{2}\right\rangle / \Delta$ t in terms of the one-dimensional power spectrum, which are normally attributed to Jokipii, are unaltered by the correction. However, as we discuss below, it is incorrect to neglect these terms containing a $\left(\mathrm{r} / \lambda_{\mathrm{c}}\right)$ at low rigidities, where $\varepsilon>1$.

K1imas and Sandri $(1971,1973 a, b)$ have also attempted to relate the diffusion tensor to observed field properties. Unlike Jokipii $(1966,1967,1968,1971,1972)$, Klimas and Sandri (1971, 1973a,b) average first over pitch angle (on assuming that the particle distribution function can be expanded in terms of Legendre polynomia1s), and then they integrate with respect to time, i.e. they integrate over $\lambda$ from 0 to $\infty$. In Appendix $B$ to this paper, we show by reversing the order of integration that Klimas and Sandri find a diffusion coefficient for propagation parallel to the mean field: 
$\kappa_{\|}=\frac{2 v^{2}}{9}\left[\int_{0}^{I} d \mu \frac{\left(1-\mu^{2}\right) \eta^{2} e^{2} v}{\lambda_{c}} \int_{0}^{\infty} d \lambda\left\{\cos (\varepsilon \lambda)\left[2 b\left(r / \lambda_{c}\right)+\rho^{2} a\left(r / \lambda_{c}\right)\right]-\rho^{2} a\left(r / \lambda_{c}\right\}\right]^{-1}\right.$

The first integra1 in (4), over $\lambda$, can be identified with $\left\langle(\Delta \mu)^{2}\right\rangle / \Delta t$ given in (l). The second integral, over $\mu$

$\kappa_{\|}=\frac{2 v^{2}}{9}\left[\int_{0}^{l} d_{\mu} \frac{<(\Delta \mu)^{2}>}{\Delta t}\right]^{-1}$

is identical with equation (4) in Jokipii (1968) or equation (46) in

Jokipii (1971), and it performs an averaging of $\left\langle(\Delta \mu)^{2}\right\rangle / \Delta t$ over pitch angle when the particle distribution function is expressed as an expansion in Legendre polynomials. The full expressions for $k_{\|}$and $<(\Delta \mu)^{2}>/ \Delta$ t due to Klimas and Sandri $(1971,1973 a, b)$ are then identica1 with the corresponding corrected results due to Jokipii (1966, 1968, 1971). However, K1imas and Sandri $(1971,1973 a, b)$ have analyzed these integrals (e.g. (4)) in more detail than Jokipii (1966, 1971) and have been able. to show formally that within the context of the quasi-linear approximation a parallel diffusion coefficient exists and propagation parallel to the mean field can be approximated by a Markovian process. Approximations to the Integral for $\left\langle(\Delta \psi)^{2}>/ \Delta t\right.$

The integral in (1) is quite complicated and therefore is probably not of mach practical value unless it can be simplified by further approximations. At high energies, where $\varepsilon<<1$, simple approximations to (1) are possible and most theories agree that the resulting diffusion coefficient has a rigidity-squared dependence (e.g. Jokipii, 1966; 
K1imas and Sandri, 1971). At low energies, where $\epsilon>>1$, valid approximations to (1) are more difficult to obtain. However, it is in this regime that it is most important to know $\left\langle\left(\Delta_{\mu}\right)^{2}\right\rangle / \Delta t$ since in the interplanetary medium galactic cosmic rays that experience significant modulation and most solar cosmic rays should satisfy $€>>1$. Jokipii (1966) suggested that when $\varepsilon>>1$ the integrand in (1) could be expanded in powers of $1 / \epsilon$. To lowest order, then, the terms containing $a\left(r / \lambda_{c}\right)$ were neglected and $b\left(r / \lambda_{c}\right)$ was evaluated at $r / \lambda_{c}=\mu \lambda$ or (I) becomes

$\frac{\left\langle(\Delta \mu)^{2}>\right.}{\Delta t}=\frac{2\left(1-\mu^{2}\right) \eta^{2} \varepsilon^{2} v}{\left.\lambda_{c} \mu\right\}} \int_{0}^{\infty} \mathrm{d} \lambda^{\prime} \cos \left(\frac{\varepsilon \lambda^{\prime}}{\mu}\right) \mathrm{b}\left(\lambda^{\prime}\right)$

The integral in (6) is simply proportional to the one-dimensional power spectrum of fluctuations in the component of the field perpendicular to the mean field direction, evaluated at the resonant wave number $\mathrm{k} \lambda_{\mathrm{c}} \approx \epsilon / \mu$, or

$\frac{<(\Delta \mu)^{2}>}{\Delta t} \simeq \frac{2 \pi\left(1-\mu^{2}\right) \eta^{2} \varepsilon^{2} v}{\lambda_{d}^{3}|\mu|} P_{\perp}\left(k=1 / \mu r_{g}\right)$

A similar expansion is implied in the work of Hall and Sturrock (1967) (cf. Equation 3.11 in their paper).

This approximation technique, however, is unlikely to be valid except for certain special forms of the correlation function (not the is otropic case considered here). By neglecting terms containing $a\left(r / \lambda_{c}\right)$ in (1) and evaluating $b\left(r / \lambda_{c}\right)$ at $r / \lambda_{c}=\mu \lambda$, we are in effect making a guiding center approximation. We evaluate the correlation function only at the particle's guiding center, or equivalently we 
assume that knowledge of the field at this point is sufficient to determine the field as seen by the particle. This approximation should work, however, only if the field varies slowly on the scale of a particle's gyro-radius. Yet in our final answer $(6)$ and $(7),\left\langle\left(\Delta_{\mu}\right)^{2}\right\rangle / \Delta t$ is determined by a resonant interaction in which a particle responds only to fluctuations that have a scale-size on the order of a gyro-radius. We can see the difficulty with this approximation by examining (1). For large $\varepsilon$, the $\cos (\epsilon \lambda)$ in the term $b\left(r / \lambda_{c}\right) \cos (\epsilon \lambda)$ varies rapid1y with $\lambda$ between positive and negative values. Thus, as the integrand adds to form the integra1, considerable cancellation will occur between these positive and negative parts. To determine $\left\langle\left(\Delta_{\mu}\right)^{2}\right\rangle / \Delta t$, then, we must know the integrand to higher accuracy than results from performing an expansion in powers of $1 / \epsilon$ and keeping only the first few terms. Mathematically this is a common problem where a valid asymptotic expansion of the integrand does not necessarily lead to a valid asymptotic expansion of the integral.

Jokipii (1972) has shown that there are special cases of the correlation function for which (6) and (7) are valid. If the field fluctuations vary effectively only with distance along the mean field direction, then the correlation length perpendicular to this direction is essentially infinite. In this case our objections to the guiding center approximation used in deriving (6) and (7) no longer hold; since now knowledge of the field at the guiding center is sufficient to specify the field as seen by the particle. It is debatable, however, as to whether actual interplanetary fluctuations have such an anisotropic correlation function. 
In general, then, it does not appear that we can determine $\left\langle(\Delta \mu)^{2}\right\rangle / \Delta t$ in the limit of large $\varepsilon$ by making a simple approximation to the integrand in (1), and we must thus apply numerical and analytic techniques to a study of the complete integral. We use in these studies a fairly general form of the correlation function, which results in power spectra that are in rough agreement with observed spectra. We take

$a\left(r / \lambda_{c}\right)=\frac{1}{6 \lambda_{c}^{2} \Gamma\left(\nu-\frac{1}{2}\right)}\left(\frac{r}{2 \lambda_{c}}\right)^{\nu-3 / 2} K_{\nu-3 / 2}\left(r / \lambda_{c}\right)$

and

$b\left(r / \lambda_{c}\right)=\frac{2}{3 \Gamma\left(\nu-\frac{1}{2}\right)}\left(\frac{r}{2 \lambda_{c}}\right)^{\nu-\frac{1}{2}}\left[K_{\nu-1 / 2}\left(r / \lambda_{c}\right)-\left(\frac{r}{2 \lambda_{c}}\right) K_{\nu-3 / 2}\left(r / \lambda_{c}\right)\right]$

where $\Gamma(z)$ is the gamma function and $K_{\nu}(z)$ is a modified Besse 1 function of the third kind and index $\nu$. By applying the appropriate Fourier cosine transforms to (8) and (9) (see Batchelor, 1957), we find that the corresponding one-dimensional power spectrum for fluctuations in the field component parallel to the mean field direction is given by $\mathrm{P}_{\|}(\mathrm{k})=\frac{\Gamma(\nu) \lambda_{c}}{3 \sqrt{\pi} \Gamma\left(\nu-\frac{1}{2}\right)}\left(1+\left(\mathrm{k} \lambda_{c}\right)^{2}\right)^{-\nu}$

and the corresponding one-dimensional power spectrum for fluctuations perpendicular to this direction is given by

$P_{\perp}(k)=\frac{P_{\|}(k)}{2}\left(1+\frac{2 \nu\left(k \lambda_{c}\right)^{2}}{1+\left(k \lambda_{c}\right)^{2}}\right)$

We must choose $\nu$ here to be greater than $\frac{1}{2}$ so that there is finite energy in the field fluctuations (we could choose $\nu<\frac{1}{2}$ and impose a 
cutoff in the power spectra at high frequencies without altering our results). However, since we can choose $v$ within the range, e.g. $1 / 2<\nu<3 / 2$ these bower spectra can be adjusted to give a good fit to observed spectra (cf. Jokipii and Coleman, 1968; Sari and Ness, 1969, 1970).

Us ing the $a\left(r / \lambda_{c}\right)$ and $b\left(r / \lambda_{c}\right)$ given in (8) and (9) respectively, we have integrated (1) numerically for a wide range of values of $\varepsilon, \nu$ and $\mu$. In the regime $\varepsilon>1$, we find that, to a good approximation (for. $\mu>0)$

$$
\frac{<(\Delta \mu)^{2}>}{\Delta t} \approx \frac{2 \pi\left(1-\mu^{2}\right) \eta^{2} \varepsilon^{2} v}{\lambda_{c}^{2}} P_{\perp}\left(k=1 / \mu r_{g}\right)
$$

where $P_{\perp}(k)$ is given in (11). That is, the actua $1<\left(\Delta_{\mu}\right)^{2}>/ \Delta$ for this correlation function differs from $\left\langle\left(\Delta_{\mu}\right)^{2}>/ \Delta t\right.$ given in (7) (due to Jokipii (1966, 1971)) only by a factor of the ratio of $\left\langle\left(\Delta_{\mu}\right)^{2}\right\rangle / \Delta t$ that results from our numerical calculations to $\left\langle(\Delta \mu)^{2}\right\rangle / \Delta t$ that results from (12) versus $\mid \mu$, for $\epsilon$ in the range $I \leq \epsilon \leq 10$ and $\nu$ in the range $3 / 4 \leq \nu \leq 5 / 4$. As can be seen in this figure to a good approximation this ratio simply equals $\mu,(\mu \neq 0)$.

For this general correlation function, then, the answer for $\left\langle\left(\Delta_{\mu}\right)^{2}\right\rangle / \Delta$ t due to Jokipii $(1966,1971)$ is numerically not significantly different from the actual answer, at all except very small values of $\mu$. It must be emphasized, however, that to a great extent the success of (7) is fortuitous. The function $b\left(r / \lambda_{c}\right)$ evaluated at the full $r / \lambda_{c}$ given in (3) is frequently an order of magnitude larger than $b\left(r / \lambda_{c}\right)$ evaluated simply at the guiding center $r / \lambda_{c}=\mu \lambda$. This result disproves the conjecture by Jokipii (1971) that higher order corrections to $b(\mu \lambda)$ 
can be neglected for $\nu<1$. The terms $a\left(r / \lambda_{c}\right)$, which are neglected in Jokipii's approximation, are actually of the same order of magnitude as the full $b\left(r / \lambda_{c}\right)$ terms, but are of opposite sign. The various terms in (1) simply add in a complicated manner to form the $\left\langle(\Delta \mu)^{2}\right\rangle / \Delta t$ which is given in (12)。

We have also studied (1) analytically. In Appendix $C$ to this paper we derive the exact analytic expression for the $\left\langle(\Delta \mu)^{2}\right\rangle / \Delta t$ that results from using the correlation function given in (8) and (9). In the limit that $\epsilon^{2} / \mu^{2}>>1$ this expression reduces to

$$
\begin{aligned}
\frac{\left\langle(\Delta \mu)^{2}>\right.}{\Delta t} & =\frac{4}{3} \frac{\Gamma(\nu+3 / 2)}{\Gamma(\nu-1 / 2)} \frac{\eta^{2} v \varepsilon^{2}}{\lambda_{c}}\left(\frac{\varepsilon}{\mu}\right)^{-2 \nu} . \\
& \cdot\left\{\sum_{m=1}^{\infty} m^{-2 \nu}[g(m, \nu, y)\right. \\
& \left.\left.+\frac{\left(1-\mu^{2}\right)}{2 \mu^{2}}[g(m+1, \nu, y)+g(m-1, \nu, y)]\right]\right\}
\end{aligned}
$$

where

$$
\begin{aligned}
g(m, v, y) & =y^{m} \frac{\Gamma(m+1 / 2) \Gamma(\nu+1-m)}{\Gamma(\nu+3 / 2) \Gamma(2 m+1)}{ }_{1} F_{z}(m+1 / 2 ; m-\nu, 2 m+1 ; y) \\
& +y^{\nu+1} \frac{\Gamma(m-\nu-1)}{\Gamma(\nu+2+m)}{ }_{1} F_{z}(\nu+3 / 2 ; \nu+2+m, \nu+2-m ; y)
\end{aligned}
$$

and $\mathrm{y}=\mathrm{m}^{2}\left(1-\mu^{2}\right) / \mu^{2}$. The function ${ }_{1} \mathrm{~F}_{\mathrm{Z}}(\alpha ; \beta, \gamma ; \mathrm{y})$ is a generalized hypergeometric function. It can be seen in Appendix $C$ that the sum over $m$ in (13) is a sum over harmonics of the resonant wavenumber $\mathrm{k} \lambda_{c}=\epsilon / \mu$. The $\varepsilon$-dependence of (13) is particularly simple and has the same functional form as the $\varepsilon$-dependence given in (12)(for large $\varepsilon$ ). 
Although we can verify (12) numerically only for $1 \leqslant \epsilon \leqslant 10$, we can conclude by using (13) that (12) should hold for arbitrarily large values of $\epsilon$. The dependence of (13) on $\mu$ and $\nu$ is contained in the complicated summation over hypergeometric functions; a summation that does not appear to simplify in any meaningful way. To the best of our knowledge, these generalized hypergeometric functions are not special cases which reduce to simpler functions, nor does it appear 1ikely that the hypergeometric functions can be combined in any simple manner. For example the two hypergeometric functions which appear in $g(m, v, y)(14)$ are linear independent solutions to the same differential equation. Of course, we can always calculate the hypergeometric functions in (13) and (14) by using numerical techniques. Upon summing typically over the first ten harmonics, we can verify that in the range $3 / 4 \leqslant \nu \leqslant 5 / 4$ and $\mu>0,\left\langle(\Delta \mu)^{2}>/ \Delta t\right.$ given in (13) is well approximated by $\left\langle(\Delta \mu)^{2}\right\rangle / \Delta t$ given in (12). The extra factor of $: \mu \mid$ in (12) as compared to (7) can have a significant effect on the pitch angle averaged diffusion coefficient in certain averaging schemes. Recently, Earl (1973) has argued that $\left.<(\Delta u)^{2}\right\rangle / \Delta t$ should be averaged over pitch angle to form $k_{\|}$by use of the formila

$K_{\|}=v^{2} \int_{-1}^{1} \mu^{\prime}\left[\int_{0}^{\mu^{\prime}} \frac{\left(1-\mu^{2}\right) d \mu}{\left\langle(\Delta \mu)^{2}\right\rangle / \Delta t}\right] d \mu^{\prime}$

rather than by use of (5). The averaging scheme in (15) was originally suggested by Jokipii (1966). However, as can be seen by substituting (12) into (15), for any $\nu \geq 1 / 2$, the first integral in (15) will tend to diverge at its lower limit, $\mu=0$. That is, $k_{\|}$as determined from (15) 
does not appear to exist for any power spectra which have the general form given in (10) and (11) and which have finite energy in the field fluctuations. We should perhaps be skeptica1 then about this averaging technique. More probably, we should be skeptical about the ability of our theory to adequately treat the behavior of particles with pitch angles near $90^{\circ}(\mu \rightarrow 0)$. In quasi-linear theory, particles resonate only with fluctuations at wavenumber $k=1 / \mu \mathrm{rg}$. As $\mu \rightarrow 0$, the resonant wavenumber tends to infinity, $P_{\perp}\left(k=1 / \mathrm{ur}_{\mathrm{g}}\right) \rightarrow 0$ and thus $\left\langle(\Delta \mu)^{2}>/ \Delta t \rightarrow 0\right.$. (exactly at $\mu=0,\left\langle(\Delta \mu)^{2}>/ \Delta t \neq 0\right.$, as we shall discuss). However, if we were to improve quasi-linear theory with, for example, some resonance broadening technique (Duprey, 1966; Weinstock 1969; Jones et a1., 1973; Völk, 1973) then we should expect that $\left\langle(\Delta \mu)^{2}\right\rangle / \Delta t$ would remain non-zero as $\mu \rightarrow 0$ and the divergence in (15) disappears. The Behavior of $\left\langle(\Delta \mu)^{2}\right\rangle / \Delta t$ at $\mu=0$

Finally, we comment that theories which use the quasi-linear and adiabatic approximations for isotropic turbulence predict neither $<(\Delta \mu)^{2}>/ \Delta t=0$ at $\mu=0($ e.g. Jokipii, 1971), nor a continuous divergence in $\left\langle(\Delta \mu)^{2}\right\rangle / \Delta t$ for small $\mu$ (Hasselmann and Wibberenz, 1968). In Appendix $D$ to this paper, we show that for an arbitrary isotropic correlation function, to lowest order $1 / \epsilon$, (1) predicts that

$\frac{<(\Delta \mu)^{2}>}{\Delta t}=\frac{\pi \pi^{2} v}{2} \delta(\mu) \int_{0}^{\infty} \mathrm{dkkP}_{\|}(\mathrm{k}) \quad$ at $\mu=0$

where $\delta(\mu)$ is a delta function.

We can obtain an expression valid for all s by following the techniques outlined by Hasselmann and Wibberenz (1968) where the perturbation 
magnetic field rather than the correlation tensor is expressed as a Fourier transform. Hasselmann and Wibberenz obtain an incorrect result because of an error when integrating over the wave-number phase angle (Völk, et al., 1973). In Appendix E to this paper we apply these techniques correctly, and obtain for $\mu=0$ :

$$
\frac{<(\Delta \mu)^{2}>}{\Delta t}=\frac{\pi(\epsilon \eta)^{2}}{2 \lambda_{c}{ }^{2}} \delta(\mu) v \quad \int_{0}^{\infty} d k P_{\|}(k)\left[3 \frac{d}{d k} g\left(k \lambda_{c} / \epsilon\right)+k \frac{d^{2}}{d k^{2}} g\left(k \lambda_{c} / \epsilon\right)\right]
$$

where

$g(\mathrm{x}) \equiv \mathrm{J}_{1}^{2}(\mathrm{x})-\mathrm{J}_{0}(\mathrm{x}) \mathrm{J}_{2}(\mathrm{x})$

and the $\mathrm{J}_{\mathrm{m}}(\mathrm{x})$ are ordinary Besse1 functions of the first kind.

We note that (16) and (17) do not describe a resonant interaction, but rather a particle responds here to fluctuations at a11 wavenumbers. As a particle spirals along its orbit it will of course experience gradients in the field due to all fluctuations, and it will have its pitch angle altered as a result of these gradients. In quasi-linear theory, however, the effects of these gradients at one point in a particle's orbit will in general be cancelled by the effects of the gradients at some other point further down the field, so that for $\mu \neq 0$ $<(\Delta \mu)^{2}>/ \Delta t \rightarrow 0$ as $\Delta t \rightarrow \infty$. Of course, the one exception to this cancellation comes from fluctuations at the resonant wavenumber, which can give rise to an accumulated change in the pitch angle, i.e. $\left\langle\left(\Delta_{\mu}\right)^{2}\right\rangle / \Delta t$ remains non-zero as $\Delta t \rightarrow \infty$. For $\mu=0$, however, quasi-1inear/adiabatic theory requires that a particle simply remains in one position along the mean field for infinite time. A particle, then, experiences the field gradients only at this one point, no cancellation of the type described 
above will occur for any wavenumber fluctuation, and $\left\langle(\Delta \mu)^{2}\right\rangle / \Delta$ t tends to infinity according to (16) and (17). We note further that this deltafunction at $\mu=0$ will not remove the divergences in (15) that occur in quasilinear theory; rather this delta function makes the integral poorly defined. Also, this delta-function will not occur in Jokipii's special case (Jokipii, 1972) where the correlation length perpendicular to the mean field direction is infinite. A particle with $\mu=0$ experiences a net change in the random field component perpendicular to the mean field direction during its circular orbit, only if the random field along the mean field direction is changing with distance. That is, for a $\mu=0$ particle to be affected by the random field on the average, $P_{\|}(k)$ must not be identically equal to zero, which is the case in these infinite correlation length models.

We do not wish to imply that this delta-function should necessarily be a part of a physically realistic derivation of $\left\langle\left(\Delta_{\mu}\right)^{2}\right\rangle / \Delta t$. However, the physics that gives rise to this delta function (i.e. changes in field magnitude, $\left.P_{\|}(k) \neq 0\right)$ must be taken into proper account.

\section{Concluding Remarks}

We have shown that despite apparent differences most theories which are based on the quasi-linear and adiabatic approximations yield the same basic integral for $\left\langle\left(\Delta_{\mu}\right)^{2}\right\rangle / \Delta t$ for the case of homogeneous, isotropic field fluctuations. For example, the theories due to Jokipii (1966, 1971) and to Klimas and Sandri (1971, 1973a,b) yield the same integral. We have also shown, however, that this integral in most cases has been evaluated incorrectly in the past. For values of $\mu$ near unity, these 
previous evaluations fortuitously yield values of $\left\langle\left(\Delta_{\mu}\right)^{2}\right\rangle / \Delta t$ close to the actual values. As $\mu \rightarrow 0$, however, the errors in previous evaluations become more significant, and at $\mu=0$ the actual $<(\Delta \mu)^{2}>/ \Delta t$ contains a delta-function which to date has been overlooked.

We should of course be dubious as to whether the quasi-linear and adiabatic approximations can be applied for dealing with particles at $\mu$ near zero. As a result, the corrected quasi-1inear/adiabatic theory presented in this paper is not expected to be a physically realistic theory for all $\mu$ particles. With a small change in field strength small $\mu$ particles can reverse direction, in violation of the quasi-1inear approximation. These particles also move relatively slowly along a correlation length, and thus there is no clear separation between the time-scale for a 'scattering' of a particle and the relaxation time of the particle distribution function, as is required for the adiabatic approximation to hold. No doubt for particles with small $\mu$, quasilinear/adiabatic theory should be modified, and replaced by a theory that treats the particle trajectories in a more realistic manner.

Due caution, however, should be exercised in making any corrections to quasi-1inear/adiabatic theory. We note in particular that none of the difficulties in evaluating $\left\langle\left(\Delta_{\mu}\right)^{2}\right\rangle / \Delta$ that we have pointed out in this paper arise in models where the correlation length perpendicular to the mean field is essentially infinite (in at least one direction). Such models have been considered recently in some detail by, e.g., Jokipii (1972, 1973), Jones, et a1. (1973), Völk (1973), and Völk, et a1., (1973). 
In any physically realistic model, however, where $\mathrm{P}_{\|}(\mathrm{k})$ is not identically zero, the corrections pointed out in this paper are expected to be important. In particular, quasi-linear/adiabatic theory will predict a delta-function in $\left\langle(\Delta \mu)^{2}\right\rangle / \Delta t$ at $\mu=0$. Any modifications to quasi-linear/adiabatic theory that are developed by using one of these infinite correlation length models will be hard pressed to deal effec. tively with this delta-function. As a result, such modifications are of doubtful importance for the development of a physically realistic theory for the behavior of particles with $\mu$ near zero.

We note also that current quasi-linear/adiabatic theory is not expected to yield accurate pitch angle averaged diffusion coefficients $\left(K_{\|}\right)$even though the inadequacies in the current theories may occur only in a narrow range of pitch angles around $\mu=0$. We saw above that the extra factor of $\mu$ that we found in $\left\langle(\Delta \mu)^{2}\right\rangle / \Delta t$ caused one of the averaging schemes for forming $\kappa_{\|}$(cf. Equation (15)) to diverge as $\mu \rightarrow 0$. It can also be shown that for reasonable power spectra, the other available averaging scheme (Equation (5)) is dominated by the contribution from the delta-function, at low rigidities (K1imas and Sandri, 1973c). In conclusion, then, no complete theory appears to be available at this time for accurately determining cosmic-ray diffusion coefficients from observed properties of the interplanetary magnetic field. 


\section{References}

Batchelor, G. K., 1956, The Theory of Homogeneous Turbulence (Cambridge: Cambridge University Press).

Duprey, T. H., 1966, Phys. Fluids, 9, 1773

Earl, J. A., 1973, Ap..J., 180, 227.

Gradshteyn, I. S., and Ryzhik, I. M., 1965, Table of Integrals, Series, and Products (New York: Academic Press).

Ha11, D. E., and Sturrock, P.A., 1967, Phys. Fluids, 10, 2620.

Hasselmann, K., and Wibberenz, G., 1968, Z. für Geophysik, 34, 353.

Jokipii, J。R. 1966, Ap. J., 146, 480 .

—. 1967 , ibid, 149, 405.

1968, 내id, $152,671$.

1971, Rev. Geophys. and Space Phys., 9, 27.

1972, Ap. J., 172, 319 .

- 1973 , ibid, 182, 585.

Jokipii, J. R., and Coleman, Jr., P.J., 1968, I. Geophys. Res., 73, 5495. Jones, F. C., Kaiser, T. B., and Birmingham, T. J., 1973, Phys. Rev.

Phys. Rev. Letters, 31, 485 .

Klimas, A. J., and Sandri, G., 1971, Ap. J., 169, 41. - $1973 a$, ibid, $180,937$. - 1973b, ibid, $184,955$.

Klimas, A. J., and Sandri, G., 1973c, Conf. Papers, 13th Inter. Conf. Cosmic Rays, $\underline{3}, 659$.

Sari, J. W., and Ness, N. F., 1969, Solar Phys., 8, 155.

Völk, H. J., 1973, preprint.

Völk, H. J., Morfil1, G., Alpers, W., 1973, preprint. Weinstock, J., 1969, Phys. Fluids, 12, 1045. 


\section{Appendix A}

Following Jokipii (1966), we calculate $\left\langle\left(\Delta_{\mu}\right)^{2}\right\rangle / \Delta t$ by using the Lorentz force equation

$\frac{\mathrm{dv}}{\mathrm{dt}}=\frac{\mathrm{q}}{\mathrm{mc}}\left(\underline{\mathrm{v}}_{\perp} \times \underline{B}_{\perp}\right)$

Here $\underline{v}_{\|}$and $\underline{v}_{1}$ are particle velocity parallel and perpendicular to the mean field direction, respectively; $q$ is particle charge; $c$, the speed of light, and $\mathrm{m}$, the relativistic particle mass. The magnetic field perpendicular to the mean field is considered to have a random component which is denoted by $B_{1}$ and which has components $B_{x}$ and $B_{y}$ in a Cartesian coordinate system with $z$-axis along the mean field. 'The average value of $\underline{B}_{1},\left\langle\underline{B}_{1}>\right.$, is zero. (Angular brackets hereafter denote ensemble average .)

To make ready use of (A.1) we must make the so-called quasi-linear approximation. We assume that the interaction between the particles and the random field is sufficiently weak so that to the lowest order of interest a particle executes an unperturbed helix about the mean field while interacting with the random field. Then, $\underline{v}_{1}$ on the right side of (A.1) is given simply by

$\underline{v}_{\perp}=v_{\perp}\left(\hat{e}_{y} \cos \omega t-\hat{e}_{x} \sin \omega t\right)$

where $\hat{e}_{x}$ and $\hat{e}_{y}$ denote unit vectors in the $x$ and $y$ directions, respectively; $v_{\perp}$ is constant, independent of time; and, $\omega=q^{B} / m c$ is the gyro-frequency of a particle in the mean field $\mathrm{B}_{0}$. According to Klimas and Sandri (1973a) the quasi-linear approximation should hold for $\varepsilon \leqslant 1$ provided that $e^{2} \eta^{2}<<1$, where $\epsilon$ is the ratio of the correlation length for field fluctuations $\left(\lambda_{c}\right)$ to a particle's gyro-radius, and $\eta$ is the 
ratio of the mean square field strength to the mean field strength squared. At low rigidities, where $e \geq 1$, Klimas and Sandri (1973a) argue that the criterion for the validity of the quasi-linear approximation is on1y that $\eta^{2}<<I$, provided that the particles are of sufficiently small pitch angle so that they do not mirror.

By using (A.2), the mean square change in a particle's pitch angle which occurs in a time interval 0 to $\Delta t$ can be obtained simply by integrating (A.1) twice, or

$$
\begin{aligned}
\left\langle(\Delta \mu)^{2\rangle}\right\rangle & =\frac{\left\langle\left(\Delta v_{\|}\right)^{2}\right\rangle}{v^{2}} \\
& =\frac{\omega^{2} v_{\perp}^{2} \eta^{2}}{v^{2}} \int_{0}^{\Delta t} d t \int_{-t}^{\Delta t-t} d t^{\prime}\left[\cos \omega t \cos \omega t^{\prime} C_{x x}\left(t, t^{\prime}\right)\right. \\
& +\left(\cos \omega t \sin \omega t^{\prime}+\sin \omega t \cos \omega t^{\prime}\right) C_{x y}\left(t, t^{\prime}\right) \\
& \left.+\sin \omega t \sin \omega t^{\prime} C_{y y}\left(t, t^{\prime}\right)\right]
\end{aligned}
$$

Here, we have replaced $\left\langle\mathrm{B}_{i}(t) \mathrm{B}_{\mathrm{j}}{ }^{\prime}\left(\mathrm{t}^{\prime}\right)\right\rangle$ with the corresponding components of the two-point correlation tensor for field fluctuations.

$C_{i j}\left(\underline{r}\left(t, t^{\prime}\right)\right)=\frac{\left\langle B_{i}(\underline{R}) B_{j}\left(\underline{R}+\underline{r}\left(t, t^{\prime}\right)\right)\right\rangle}{B_{o}{ }^{2} \eta^{2}}$

The field fluctuations are assumed to be homogeneous, so that $\mathrm{C}_{i j}$ is independent of some arbitrary position vector $\underline{R}$ and a function only of $\underline{r}\left(t, t^{\prime}\right)$, the vector separating a particle's position at time $t$ and at $t^{\prime}$.

In the next step in our derivation we change variables in (A.3) from $t$ and $t^{\prime}$ to $t$ and $\tau=t^{\prime}-t$, and then we reverse the order of integration. After a considerable amount of tedious but straightforward algebra, we find that (A.3) becomes 


$$
\begin{aligned}
<(\Delta \mu)^{2}> & =\frac{\omega^{2} v_{\perp}^{2} T^{2}}{v^{2}} \int_{0}^{\Delta t} \mathrm{~d} \tau\left\{\int _ { 0 } ^ { \Delta t - \tau } \left[\frac{\cos \omega \tau}{2}\left(C_{x x}+C_{y y}\right)\right.\right. \\
& +\left(\cos \omega_{\tau} \cos 2 \omega t-\sin \omega_{\tau} \sin 2 \omega t\right)\left(\frac{C_{x x}-C_{y y}}{2}\right) \\
& \left.+\left(\sin \omega_{T} \cos 2 \omega t+\cos \omega_{\tau} \sin 2 \omega t\right) C_{x y}\right] \\
& +\int_{T}^{\Delta t} d t\left[\frac{\cos \omega_{T}}{2}\left(C_{x x}+C_{y y}\right)\right. \\
& +\left(\cos \omega_{T} \cos 2 \omega t+\sin \omega_{\tau} \sin 2 \omega t\right)\left[\frac{C_{x x}-C_{y y}}{2}\right] \\
& \left.\left.+\left(\cos \omega_{\tau} \sin 2 \omega t-\sin \omega_{\tau} \cos 2 \omega t\right) C_{x y}\right]\right\}
\end{aligned}
$$

If we further assume that the fluctuations are isotropic, then

$$
C_{i j}(\underline{r})=b(r) \delta_{i j}+a(r) r_{i} r_{j}
$$

where $a(r)$ and $b(r)$ are arbitrary even functions of the scaler separation $r$, which in turn is a function only of $\tau$ given by

$$
\begin{aligned}
\mathrm{r}^{2}(\tau) & =\mathrm{v}_{\|}{ }^{2} \tau^{2}+\frac{2 \mathrm{v}_{\perp}^{2}}{\omega^{2}}\left(1-\cos \omega_{\tau}\right) \\
& =v_{\|}{ }^{2} \tau^{2}+\rho^{2}(\tau) \lambda_{c}{ }^{2}
\end{aligned}
$$

The first integral in (A.5), then, can be done exactly; it is independent of $a(r(T))$ and $b(r(T))$ and involves integrating only simple trigonometric functions. The resulting terms are either linear in $(\Delta t-\tau)$ or proportional to $1 / \omega$. These latter terms can be neglected and we find that

$$
\begin{aligned}
<\left(\Delta_{\mu}\right)^{2}>= & \frac{\omega^{2} v_{1}^{2} \eta_{T}^{2}}{v^{2}} \int_{0}^{\Delta t} d \tau \\
& (\Delta t-\tau)\left[\cos \omega_{\tau}\left[a(r(\tau)) \rho^{2} \lambda_{c}+2 b(r(\tau))\right]-a(r(\tau)) \rho^{2} \lambda_{c}{ }^{2}\right]
\end{aligned}
$$


Finally, we make the so-cal led adiabatic approximation. The integrand in (A.8) is assumed to fall-off with a characteristic time $\tilde{\tau}$, so that for $\Delta t>>\tilde{\tau}$, we can approximate $\Delta t-\tau$ as $\Delta t$ and extend the upper limit of integration to infinity. In terms of a dimensionless time $\lambda=v_{T} / \lambda_{c},(A .8)$ then reduces to (1). 


\section{Appendix B}

In this appendix we show that the result of K1imas and Sandri $(1971,1973 a, b)$ for $\kappa_{\|}$can be expressed in the form given in equation (4) of the text.

Upon replacing Klimas and Sandri's characteristic length $\lambda_{\mathrm{P}}$ with our $\lambda_{c}\left(\lambda_{p}=\sqrt{ } \lambda_{c} / 6\right)$, we see from equation B.4 in Klimas and Sandri (1973b) that these authors find that

$x_{\|}=\frac{1}{3} \frac{v \lambda_{c}}{\eta^{2}} \frac{1}{\epsilon^{2} \Delta_{\|}}$

By using equation B.3 in this same paper, and equation 3.19 in Klimas and Sandri (1971), we find that

$\Delta_{\|}=\int_{0}^{\infty} \mathrm{d} \lambda\left[2 \mathrm{~J}_{1}(\epsilon, \lambda) \cos (\epsilon \lambda)-8 \lambda^{2} \mathrm{I}_{1}(\epsilon, \lambda)\left(\frac{\cos (\epsilon \lambda)-1}{\epsilon \lambda}\right)^{2}\right]$

where, by using equations (3.22), (3.24), (3.15), and (3.16) in this latter paper,

$J_{\perp}=\frac{3}{2} \int_{0}^{1} d \mu b\left(r / \lambda_{c}\right)\left(1-\mu^{2}\right)$

and

$I_{1}=\frac{3}{8} \int_{0}^{1} d \mu a\left(r / \lambda_{c}\right)\left(1-\mu^{2}\right)^{2}$

We note here that in Klimas and Sandri's notation, our $a\left(r / \lambda_{c}\right)$ and $b\left(r / \lambda_{c}\right)$ are $B\left(r / \lambda_{c}\right)$ and $A\left(r / \lambda_{c}\right)$, respectively. Finally by substituting (B.2), (B.3) and (B.4), from above, into (B.I), and upon reversing the order of integration, we obtain (4). 


\section{Appendix C}

In this appendix we evaluate analytically $\left\langle(\Delta,)^{2}>/ \Delta t\right.$ when the power spectrum of magnetic field irregularities is given by eqs. (10) and (11). Starting from (1) we have

$<(\Delta \mu)^{2}>/ \Delta t=\frac{\left(1-\mu^{2}\right) \eta^{2} \varepsilon^{2} v}{\lambda_{c}} I$

where

$I=\int_{0}^{\infty} d \lambda\left\{\cos \epsilon \lambda\left[2 b\left(r / \lambda_{c}\right)+a\left(r / \lambda_{c}\right) \rho^{2}\right]-a\left(r / \lambda_{c}\right) \rho^{2}\right\}$

and

$\frac{r}{\lambda_{c}}=\left[\mu^{2} \lambda^{2}+\frac{4\left(1-\mu^{2}\right)}{\epsilon^{2}} \sin ^{2}\left(\frac{\varepsilon \lambda}{2}\right)\right]^{\frac{1}{2}} \equiv\left(\mu^{2} \lambda^{2}+\rho^{2}\right)^{\frac{1}{2}}$.

Because $r$, $a$ and $b$ are such complicated functions of $\lambda,(C .2)$ cannot be integrated directly. One possible approach would be to transform $a$ and $b$ into three-dimensional Fourier-transforms of the power spectra. The functional dependence of $I$ on $r$ would be contained in terms proportional to exp $\mathbf{i} \underline{k} \cdot \underline{r}$ and no terms would be functions of $|\underline{r}|$. However, because it is not possible to write the last term in (C.2) in terms of the correlation tensor, we proceed by first transforming I into a new coordinate system in which the magnitude of $\underline{r}$ rema ins unchanged, but the components are

$\frac{r_{z}}{\lambda_{c}}=\mu \lambda, \frac{r_{x}}{\lambda_{c}}=\rho, \frac{r_{y}}{\lambda_{c}}=0$

We can now define an isotropic correlation tensor $R_{i j}(\underline{r})$ with components

$R_{i j}(\underline{r})=b\left(r / \lambda_{c}\right) \delta_{i j}+a\left(r / \lambda_{c}\right) r_{i} r_{j}$ 
so that

$$
\begin{aligned}
& \mathrm{R}_{\mathrm{xx}}=\mathrm{b}\left(\mathrm{r} / \lambda_{\mathrm{c}}\right)+\rho^{2} \mathrm{a}\left(\mathrm{r} / \lambda_{c}\right) \\
& \mathrm{R}_{\mathrm{yy}}=\mathrm{b}\left(\mathrm{r} / \lambda_{\mathrm{c}}\right)
\end{aligned}
$$

If we identify the scalar functions $a$ and $b$ in (C.5-6) with a and

$b$ in $(C .2)$, we have

$I=\int_{0}^{\infty} d \lambda\left[\cos (\epsilon \lambda)\left(R_{x x}+R_{y y}\right)-\left(R_{x x}-R_{y y}\right)\right]$

The components of the tensor $R_{i j}(\underline{r})$ can be expressed as three-dimensional Fourier-transforms of a power spectral tensor $P_{i j}$ :

$R_{i j}(\underline{r})=\int d \underline{k} P_{i j}(\underline{k}) e^{i \underline{k} \cdot \underline{r}}$

so that

$$
I=\frac{1}{2} \int_{-\infty}^{\infty} d \lambda\left\{e^{-i \epsilon \lambda}\left[\int d \underline{k}\left(P_{x x}+P_{y y}\right) e^{i \underline{k} \cdot \underline{\underline{r}}}\right]-\int d \underline{k}\left(P_{x x}-P_{y y}\right) e^{i \underline{k} \cdot \underline{r}}\right\}
$$

We now interchange the order of integration, and write

$\underline{\mathrm{k}} \cdot \underline{\mathrm{r}}=\mathrm{k}_{\mathrm{z}} \lambda_{\mathrm{c}} \mu \lambda+\frac{2 \mu^{\prime}}{\epsilon} \mathrm{k}_{\mathrm{x}} \lambda_{\mathrm{c}} \sin (\epsilon \lambda / 2)$

where $\mu^{\prime} \equiv\left(1-\mu^{2}\right)^{\frac{1}{2}}$. The integration over $\lambda$ can now be performed by making use of the following identity

$e^{i z \sin \varphi}=\sum_{m=-\infty}^{\infty} e^{i m \varphi} J_{m}(z)$

where $J_{m}(z)$ are the Bessel function of the first kind.

Therefore (C.9) becoines

$$
\begin{aligned}
& I=\pi \sum_{m}^{\infty} \int d \underline{k}\left\{\left(P_{x x}+P_{y y}\right) J_{m}\left(2 \mu{ }^{\prime} k_{x} \lambda_{c} / s\right) \delta\left[k_{z} \lambda_{c} \mu-\epsilon(1-m / 2)\right]\right. \\
& \left.-\left(P_{x x}-P_{y y}\right) J_{m}\left(2 \psi^{\prime} k_{x} \lambda_{c} / \epsilon\right) \delta\left[k_{z} \lambda_{c} \psi+m \epsilon / 2\right]\right\}^{*} .
\end{aligned}
$$


Note that $P_{x x}$ and $P_{y y}$ are even functions of $k_{x}$, so that the sum in (C.12) is non-zero only for even $\mathrm{m}$. Therefore, after integrating over $k_{z}$, the integral can be written as

$$
I=\left.\frac{\pi}{\lambda_{c} \mu} \sum_{m=-\infty}^{\infty} \int_{-\infty}^{+\infty} d k_{x} \int_{-\infty}^{+\infty} d k_{y}\left\{P_{x x}\left(J_{2 m+2}-J_{2 m}\right)+P_{y y}\left(J_{2 m+z}+J_{2 m}\right)\right\}\right|_{k_{z}}=\frac{m s}{\mu \lambda_{c}}
$$

In genera1, the $P_{x x}$ and $P_{y y}$ are given by (Batchelor, 1956)

$P_{i j}(k)=\frac{E(k)}{4 \pi k^{4}}\left(k^{2} \delta_{i j}-k_{i} k_{j}\right)$

where

$E(k)=k^{3}\left[\frac{d}{d k}\left(\frac{1}{k} \frac{d P_{1}}{d k}\right)\right]$

and $P_{\|}$is given by eq. (10). One should note that this definition of $P_{\|}$ ensures that $a\left(r / \lambda_{c}\right)$ and $b\left(r / \lambda_{c}\right)$ are given by eqs. ( 8 ) and (9.). The integral over $k_{y}$ can now be performed with the result

$$
\begin{aligned}
I & =\frac{4 \Gamma(\nu+3 / 2) e^{2} \lambda_{c}}{3 \Gamma(\nu-1 / 2) \mu \mu^{\prime 2}} \sum_{m=0}^{\infty} m^{2} \int_{0}^{\infty} d k_{x}\left(1+\left(k_{x} \lambda_{c}\right)^{2}+\left(k_{z} \lambda_{c}\right)^{2}\right)^{-\nu-3 / 2}\left\{2 J_{2 m}\left(\frac{2 \mu^{\prime} k_{x} \lambda_{c}}{\epsilon}\right)\right. \\
& \left.+\frac{\mu^{\prime 2}}{\mu^{2}}\left[J_{z m-2}\left(\frac{2 \mu^{\prime} k_{x} \lambda_{c}}{\epsilon}\right)+J_{2 m+2}\left(\frac{2 \mu^{\prime} k_{x} \lambda_{c}}{\varepsilon}\right)\right]\right\}
\end{aligned}
$$

where we have rearranged the summation so that it extends only over $m \geq 0$. For non-zero $h$, the $m=0$ term is zero. That this term is in fact a delta-function is shown in Appendix D. (For non-zero m, each term in the sum equals zero for $\mu=0$.) We now restrict ourselves to $\mu \neq 0$ and drop the $m=0$ term. The remaining integral over $k_{x}$ can also be performed (Gradshteyn and Ryzhik, 1965) so that 


$$
\begin{aligned}
<(\Delta \mu)^{2}>/ \Delta t & =\frac{4 \Gamma(\nu+3 / 2) \eta^{2} v \varepsilon^{2}}{3 \Gamma(\nu-1 / 2) \lambda_{c}} \\
& \left\{\sum_{m=1}^{\infty} \mu m^{2}\left(\frac{\epsilon}{\mu}\right)^{2}\left(1+m^{3} \varepsilon^{2} / \mu_{0}^{2}\right)-\nu-1[g(m, \nu, y)+\right. \\
& \left.\left.\frac{\left(1-\mu^{2}\right)}{2 \mu^{2}}[g(m+1, \nu, y)+g(m-1, \nu, y)]\right]\right\}
\end{aligned}
$$

where $g(m, v, y)$ is defined by eq. (14) with

$y \equiv \frac{\left(1-\mu^{2}\right)}{e^{2}}\left(1+\mathrm{m}^{2} e^{2} / \mu^{2}\right)$

In the limit of low rigidity $(\varepsilon>>1)$, (c.17) reduces to (13) in the text. 


\section{Appendix D}

We can derive (16) by expanding $\frac{\left\langle\left(\Delta_{\mu}\right)^{2}\right\rangle}{\Delta t}$ in (I), in powers of $1 / \mathrm{s}$; i.e., in powers of the gyroradius. We have demonstrated that this procedure does not produce a rapidly converging series for the resonant interaction, but we will see here that a true power series in $(1 / \epsilon)^{2}$ can be obtained for the delta-function term. As a demonstration, the first correction to (16) will also be constructed. In Appendix E we derive this delta function term from a different point of view for all e.

The two functions, $a\left(\frac{r}{\lambda_{c}}\right)$ and $b\left(\frac{r}{\lambda_{c}}\right)$, which appear in (1) for $\frac{\left\langle\left(\Delta_{\mu}\right)^{2} \geq\right.}{\Delta \mathrm{t}}$ are not independent of each other. From $\underline{\nabla} \cdot \underline{\mathrm{B}}=0$, it follows that

$b\left(\frac{r}{\lambda_{c}}\right)=2 \int_{r / \lambda_{c}}^{\infty} \mathrm{dxx} a(x)-\left(\frac{r^{2}}{\lambda_{c}}\right) a\left(\frac{r}{\lambda_{c}}\right)$

which we substitute into (1) to give

$$
\begin{aligned}
\frac{\left\langle(\Delta \mu)^{2}>\right.}{\Delta t} & =2 \frac{\left(1-\mu^{2}\right) \eta^{2} \varepsilon^{2} v}{\lambda_{c}} \int_{0}^{\infty} \mathrm{d} \lambda\left[\cos \epsilon \lambda\left(2 \int_{r / \lambda_{c}}^{\infty} d x x a(x)-\left(\frac{r^{2}}{\lambda_{c}}\right) a\left(\frac{r}{\lambda_{c}}\right)\right)+\right. \\
& \left.-\frac{1}{\epsilon^{2}}(\cos \epsilon \lambda-1)^{2}\left(1-\mu^{2}\right) a\left(\frac{r}{\lambda_{c}}\right)\right]
\end{aligned}
$$

We now construct the Taylor Series expansions of $a\left(\frac{r}{\lambda_{c}}\right)$ and $I\left(\frac{r}{\lambda_{c}}\right) \equiv \int_{r / \lambda_{c}}^{\infty} \mathrm{dxx} a(\mathrm{x})$

in powers of $r_{0}^{2}=\frac{2}{\epsilon^{2}}(1-\cos \varepsilon \lambda)$. The results of these expansions are, 
$I\left(\frac{r}{\lambda_{c}}\right)=I(\mu \lambda)-\frac{1}{2} r_{0}^{2}\left(1-\mu^{2}\right) a(\mu \lambda)+$

$$
-\frac{1}{8} r_{0}^{4} \frac{\left(1-\mu^{2}\right)^{2}}{\mu \lambda} a^{\prime}(\mu \lambda)+\cdots
$$

and,

$$
\begin{aligned}
a\left(\frac{r}{\lambda_{c}}\right) & =a(\mu \lambda)+\frac{1}{2} r_{0}^{2}\left(\frac{1-\mu^{2}}{\mu \lambda}\right) a^{\prime}(\mu \lambda)+ \\
& +\frac{1}{8} r_{0}^{4}\left(\frac{1-\mu^{2}}{\mu \lambda}\right)\left[a^{\prime \prime}(\mu \lambda)-\frac{a^{\prime}(\mu \lambda)}{\mu \lambda}\right]+\cdots
\end{aligned}
$$

These expansions are uniform in $\mu$ and $\lambda$ for $0 \leq \mu \leq 1$, and $0 \leq \lambda \leq \infty$. Upon substituting these expansions into (D-2) we are able to construct the series,

$\frac{\left\langle(\Delta \mu)^{2} \geq\right.}{\Delta t}=\left.\frac{\left\langle(\Delta \mu)^{2} \geq\right.}{\Delta t}\right|_{0}+\left.\left(\frac{1}{\epsilon}\right)^{2} \frac{\left\langle(\Delta \mu)^{2}>\right.}{\Delta t}\right|_{2}+\left.\underset{\epsilon}{\left(\frac{1}{2}\right)^{4}} \frac{\left\langle(\Delta \mu)^{2}>\right.}{\Delta t}\right|_{4}+\cdots$

It should be noted that the coefficients in this expansion are functions of $e$; as it stands at this point, (D-6) is not a simple power series expansion in $1 / \epsilon$.

The leading term in (D-6) is given by,

$\left.\frac{\leq(\Delta \mu)^{2} \geq}{\Delta t}\right|_{0}=2 \frac{\left(1-\mu^{2}\right) \eta^{2} \varepsilon^{2} v}{\mu \lambda_{c}} \int_{0}^{\infty} \mathrm{d} \lambda \cos \left(\frac{\epsilon \lambda}{\mu}\right) b(\lambda)$

or, in terms of $P_{\perp}(k)$,

$\left.\frac{<(\Delta \mu)^{2}>}{\Delta t}\right|_{0}=\pi \frac{\left(1-\mu^{2}\right) \eta^{2} \varepsilon^{2} v}{\mu \lambda_{c}^{2}} P_{\perp}\left(k=\frac{1}{\mu r_{g}}\right)$

These are alternate expressions for Jokipii's (1966, 1971) approximation to the resonant interaction given in (6) and (7). 
The next term in (D-6) is given by,

$$
\begin{aligned}
\left.\frac{\left\langle(\Delta \mu)^{2} \geq\right.}{\Delta t}\right|_{a} & =\frac{\left(1-\mu^{2}\right) \eta^{2} \epsilon^{2} v}{\lambda_{c}} \lim _{\mathrm{T} \rightarrow \infty} \int_{0}^{\mathrm{T}} \mathrm{d} \lambda\left[\left(\mathrm{a}(\mu \lambda)+\mu \lambda \mathrm{a}^{\prime}(\mu \lambda)\right)+\right. \\
& \left.-2 \cos \left(\varepsilon \lambda \times 2 \mathrm{a}(\mu \lambda)+\mu \lambda \mathrm{a}^{\prime}(\mu \lambda)\right)+\cos (2 \varepsilon \lambda)\left(3 \mathrm{a}(\mu \lambda)+\mu \lambda \mathrm{a}^{\prime}(\mu \lambda)\right)\right]
\end{aligned}
$$

The limit on $\mathrm{T}$ is included here to ensure that the change of integration variable, from $\lambda$ to $r=\mu \lambda$, which we are about to introduce, is carried out correctly. The last two terms in the integrand of (D-9) contribute to the resonant interaction discussed fully in the main text. Thus we ignore these terms here, and study the first term which represents the leading contribution, in $1 / \epsilon$, to the delta function term. This contribution is given by,

$$
\left.\frac{\left\langle(\Delta \mu)^{2}>\right.}{\Delta t}\right|_{a}=\frac{\left(1-\mu^{2}\right) \eta^{2} \varepsilon^{2} v}{\mu \lambda_{c}} \lim _{\mathrm{T} \rightarrow \infty} \int_{0}^{\mu \mathrm{T}} \mathrm{dr} \frac{\mathrm{d}}{\mathrm{dr}}(\mathrm{r} \quad \mathrm{a}(\mathrm{r}))
$$

or

$\left.\frac{\left\langle\left(\Delta_{\mu}\right)^{2}>\right.}{\Delta t}\right|_{2}=\frac{\left(1-\mu^{2}\right) \eta^{2} \epsilon^{2} v}{\lambda_{c}} \lim _{\mathrm{T} \rightarrow \infty} \mathrm{T} a(\mu \mathrm{T})$

If $a(r) \rightarrow 0$ as $r \rightarrow \infty$, faster than $1 / r$, then, for $\mu=0, \lim _{T \rightarrow \infty} T a(\mu T)=, a(0)$. $\lim _{T \rightarrow \infty} T=\infty . \quad$ But, for $\mu \neq 0, \lim _{T \rightarrow \infty} T a(\mu T)=0 . \quad$ Furthermore, $\int_{0}^{1} d \mu \lim _{T \rightarrow \infty} T a(\mu T)=\lim _{T \rightarrow \infty} \int_{0}^{T} d r a(r)=\int_{0}^{\infty} d r a(r)$

Thus, as a function of $\mu, \lim _{T \rightarrow \infty} T a(\mu T)$ is zero for all $\mu>0$, is infinite at $\mu=0$, and can be integrated to give a finite, non-zero number, i.e. it exhibits all the properties of a delta function, or 
$\left.\frac{\left\langle\left(\dot{\Delta}_{\mu}\right)^{2} \geq\right.}{\Delta \mathrm{t}}\right|_{2}=\frac{\eta^{2} e^{2} \mathrm{v}}{\lambda_{c}} \delta(\mu) \int_{0}^{\infty} \mathrm{dr} \mathrm{a}(\mathrm{r})$

In terms of the power spectrum,

$\left.\frac{<(\Delta \mu)^{2}>}{\Delta t}\right|_{2}=\frac{\pi \eta^{2} \varepsilon^{2} \mathrm{v}}{2} \delta(\mu) \int_{0}^{\infty} \mathrm{dk} \mathrm{kP}_{\|}(\mathrm{k})$

If we once again drop the contributions to the resonant interaction in the fourth order term in (D-6), we find,

$\left.\frac{\left\langle(\Delta \mu)^{2} \geq\right.}{\Delta t}\right|_{4}=\frac{\left(1-\mu^{2}\right)^{3} \eta^{2} \varepsilon^{2} v}{\lambda_{c}} \lim _{\mathrm{T} \rightarrow \infty} \int_{0}^{\mathrm{T}} \mathrm{d} \lambda \mathrm{a}^{\prime \prime}(\mu \lambda)$

For homogeneous turbulence in continuous fields, we must have $a^{\prime}(0)=0$ (Batchelor, 1956). Therefore,

$\left.\frac{<(\Delta \mu)^{2}>}{\Delta t}\right|_{4}=\frac{\left(1-\mu^{2}\right)^{3} \eta^{2} \varepsilon^{2} v}{\lambda_{c}} \lim _{\mathrm{T} \rightarrow \infty} \mathrm{T}\left(\frac{\mathrm{a}^{\prime}(\mu \mathrm{T})}{\mu \mathrm{T}}\right)$.

Finally, using reasoning similar to that presented above for the leading contribution to the delta function term, we find,

$\left.\frac{<(\Delta \mu)^{2}>}{\Delta t}\right|_{4}=\frac{\eta^{2} \epsilon^{2} v}{\lambda_{c}} \delta(\mu) \int_{0}^{\infty} \mathrm{dr} \frac{a^{\prime}(r)}{r}$.

Since $a^{\prime}(r)$ is proportional to $r$ for small $r$, the integral in $(D-17)$ is finite. Equation (D-17) gives the $0\left(\left(\frac{1}{\varepsilon}\right)^{2}\right)$ term in a power series expansion of (D-6). Rather than continue this calculation to higher orders we present in Appendix $E$ a different procedure which gives the delta function term for all $\varepsilon$. 


\section{Appendix E}

We calculate below an alternative expression for the Fokker-Planck pitch angle scattering coefficient for isotropic turbulence and show that at $\mu=0$ it is proportiona 1 to a de1ta-function to all orders in $\varepsilon$. We again begin with the equation of motion (A.1) and Fourier transform the random component of the magnetic field. One again uses the identity (C.11) so that

$$
\begin{aligned}
& \left.<\left(\Delta_{\mu}\right)^{2}\right\rangle=\frac{\left(\varepsilon \eta \mu^{\prime}\right)^{2}}{4 \pi} \int_{0}^{\tau} \mathrm{d} \lambda \int_{0}^{\tau} \mathrm{d} \lambda^{\prime} \int \frac{\mathrm{dk}}{\mathrm{k}^{2}} \sum_{\mathrm{n}=-\infty}^{\infty} \sum_{\mathrm{m}=-\infty}^{\infty} \mathrm{e}^{i k_{\|} \lambda_{\mathrm{c}} \mu\left(\lambda-\lambda^{\prime}\right)} \\
& e^{i \epsilon\left(n \lambda-m \lambda^{\prime}\right)} e^{-i \psi(n-m)} J_{n}\left(\frac{k_{\perp} \lambda_{c} \mu^{\prime}}{\varepsilon}\right) J_{m}\left(\frac{k_{\perp} \lambda_{c} \mu^{\prime}}{\varepsilon}\right) \\
& {\left[\cos \varepsilon\left(\lambda-\lambda^{\prime}\right)-\left(\frac{k_{1}}{k}\right)^{2} \sin (\psi-\epsilon \lambda) \sin \left(\psi-\epsilon \lambda^{\prime}\right)\right]}
\end{aligned}
$$

where $\mathrm{E}(\mathrm{k})$ is given by $(\mathrm{C} .15), \mu^{\prime} \equiv\left(1-\mu^{2}\right)^{\frac{1}{2}}$ and $\psi$ is the wave-number phase angle. The integrals over $\psi, \lambda$, and $\lambda^{\prime}$ can be readily performed using the standard adiabatic approximation (cf. Appendix A) with the result

$$
\begin{aligned}
\leq \frac{\left\langle(\Delta \mu)^{2} \geq\right.}{\Delta t}= & \frac{\pi}{2 \lambda_{c}}\left(\epsilon \eta_{\mu} \mu^{\prime}\right)^{2} v \int_{0}^{\infty} d k_{\perp} k_{\perp} \cdot \int_{-\infty}^{\infty} d k_{\|} \frac{E(k)}{k^{2}} \sum_{m=-\infty}^{+\infty} \delta\left(\mu k_{\| l} \lambda_{c}+m \epsilon\right) \\
& \left\{J_{m-1}^{2}\left(\frac{k_{\perp} \lambda_{c} \mu^{\prime}}{\epsilon}\right)+J_{m+1}^{2}\left(\frac{k_{\perp} \lambda_{c \mu^{\prime}}}{\epsilon}\right)-2\left(\frac{k_{\perp}}{k}\right)^{2}\left[J_{m}\left(\frac{k_{\perp} \lambda_{c} \mu^{\prime}}{\epsilon}\right)\right]^{2}\right\} .
\end{aligned}
$$

For $\mu \neq 0$ one can readily integrate over $k_{\|}$. However, we want to investigate $(\mathrm{E} .2)$ when $\mu=0$. In this case we transform to polar coordinates and note that only the $m=0$ term in the summation is nonzero at $\mu=0$ 。 One can integrate over polar angle, leaving 
$-32-$

$\frac{\left\langle(\Delta \mu)^{2}>\right.}{\Delta t}=\frac{\pi \varepsilon^{2} \eta^{2} v}{2 \lambda_{c}^{2}} \delta(\mu) \int_{0}^{\infty} d k \frac{E(k)}{k}\left[J_{1}^{2}\left(\frac{k \lambda_{c}}{\varepsilon}\right)-J_{0}\left(k \lambda_{c} / \varepsilon\right) J_{2}\left(\frac{k \lambda_{c}}{\varepsilon}\right)\right]$. (E.3)

Using the definition of $\mathrm{E}(\mathrm{k})$ one arrives at (17). 


\section{Figure Caption-}

The ratio of the exact numerical integration of $(1)\left(\left\langle(\Delta \mu)^{2}\right\rangle / \Delta t\right)$ to the approximate result (7) due to Jokipii $\left(\left\langle\left(\Delta_{H}\right)^{2}\right\rangle / \Delta \mathrm{t}\right)_{\mathrm{J}}$ is plotted against $! \mu \mid(\mu \neq 0)$ for various values of $\varepsilon$ and $\nu$. To a good approximation the exact result is smaller than the approximate result by a factor of $|\mu|$ 

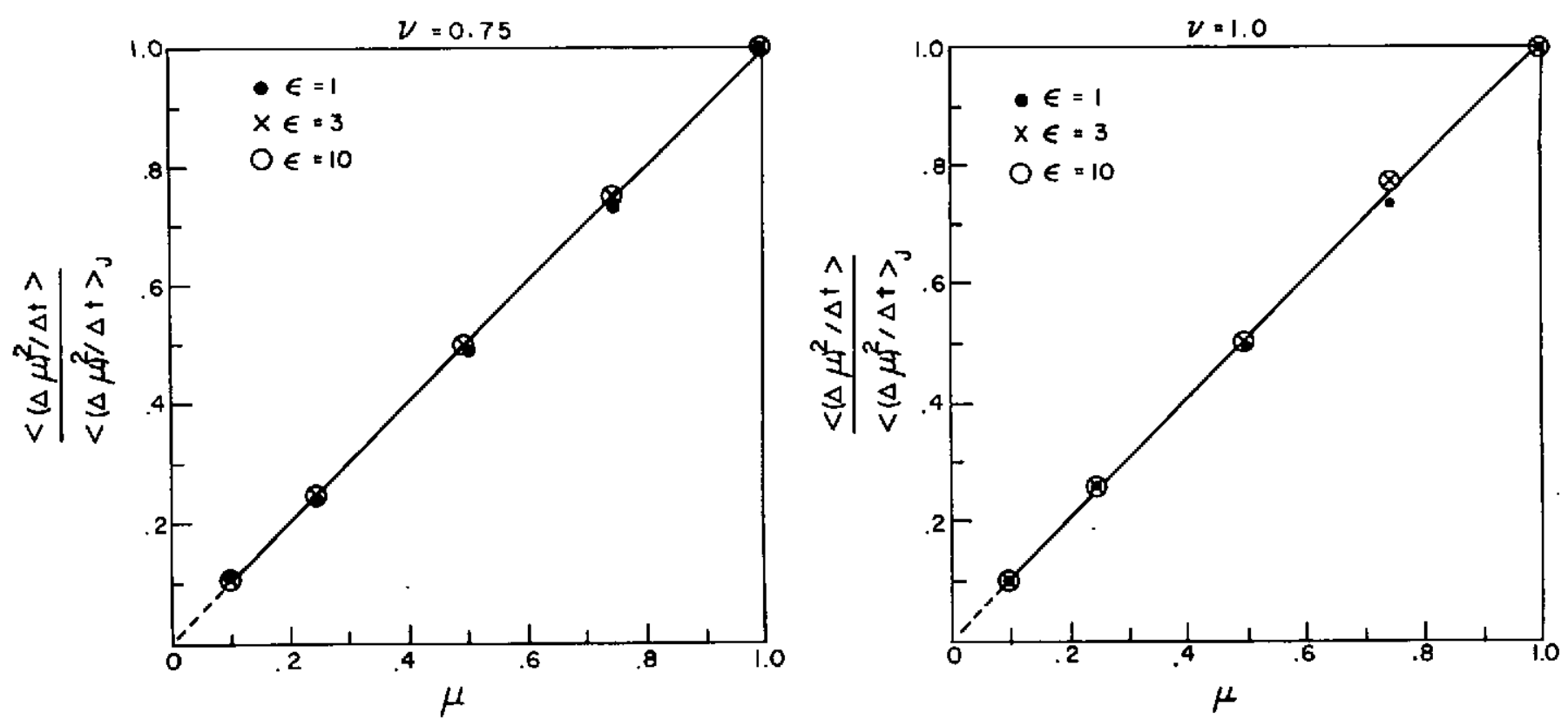

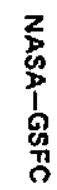

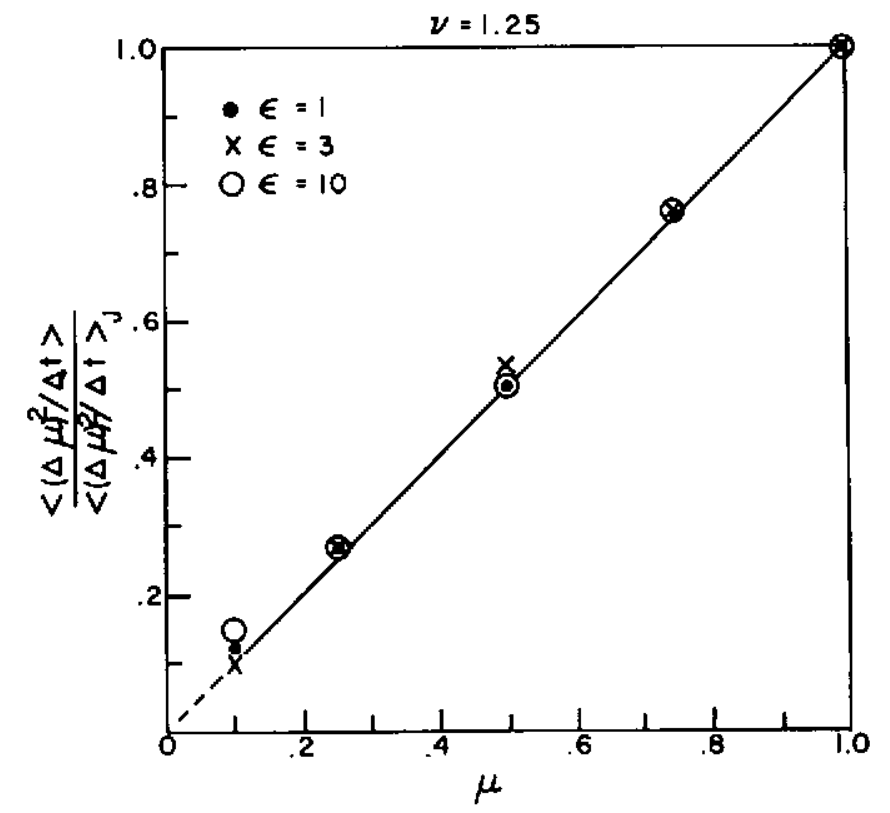

